\title{
Papillary Microcarcinoma: AN Epidemiological, Clinical, therapeutic and Prognostic Study of 35 Cases
}

\author{
Maha Hakimi, Fatimaazahra Hadid, Youssef Rochdi, Hassan Nouri, and Abdelaziz Raji
}

\section{ABSTRACT}

Papillary microcarcinoma of the thyroid is defined as cancers having a diameter equal or inferior to $10 \mathrm{~mm}$ in the anatomopathological examination; The problematic of this pathology lies in its latent character, it is, thus, much more frequent than we thought or predicted. That is why, beyond specifying the epidemiological and clinical characteristics of papillary thyroid microcarcinoma, our ultimate goal was to determine the "gold standard" for the management of this entity.

We carried out a descriptive retrospective study spread over 4 years including all cases of Papillary Microcarcinoma of the thyroid diagnosed within the department of ENT and Head and Neck Surgery of the medical center Mohammed VI in Marrakech; We collected 35 cases, and using their medical records we summarized their epidemiological, clinical and paraclinical data.

None of our patients had a normal clinical examination, the palpation of our patients showed an isolated nodule in $20 \%$ of patients, the rest of our patients had clinically bilateral noticeable nodes. $80 \%$ of which were not clinically suspicious, and only three of our patients had palpable lymph nodes which was confirmed by the ultrasound, where the major suspicious character was their hypoechoic character ( $17 \%$ were EU-TIRADS 5 ) With regard to surgical treatment, 28 of our patients $(80 \%)$ underwent total thyroidectomy as a first step, 4 patients underwent secondary totalization during the same operation after the extemporaneous examination came back positive for papillary microcarcinoma , 2 cases of remote loboisthmectomy after final histological examination showing papillary microcarcinoma, and one final case of totalization after extemporaneous examination of a superior right jugular lymph node with central necrosis. Neck dissection was performed in three cases. Postoperative outcomes were simple in all patients. Isotopic totalization by iodine 131 therapy was performed in four of our patients. Their indications were respectively: infiltrating tumor, extension to neighboring parenchyma, multifocality, lymph node metastasis. The survival rate of our series was $100 \%$. The therapeutic protocol of papillary microcarcinoma is punctuated, to this day, with controversy, and faces a conservative attitude versus a radical one, it is the surgeon's duty to distinguish between the two attitudes.

Keywords: Neck dissection, Papillary microcarcinoma, thyroid, total thyroidectomy.

\section{INTRODUCTION}

Papillary microcarcinoma of the thyroid have a rather strict histological definition demarcated by the World Health Organization in 1988 as cancers having a diameter equal or inferior to $10 \mathrm{~mm}$ in the anatomopathological examination [1]. They established, then, that microcarcinomas constitute about $30 \%$ of all papillary carcinomas and about $40 \%$ in 2003, making it the most common variant of papillary carcinoma in the United States. Similar trends have been observed in France and in many other countries of the world; so also noticed in our humble study [2]. The
Published Online: December 31, 2020

ISSN: 2593-8339

DOI: $10.24018 /$ ejmed.2020.2.6.627

\section{Maha Hakimi*}

ENT - Neck and Head surgery Department; University Medical Center Mohammed VI, Morocco.

(e-mail: mahahakimi1@gmail.com)

Fatimaazahra Hadid

ENT - Neck and Head surgery Department; University Medical Center Mohammed VI, Morocco.

(e-mail: fz.hadid@gmail.com)

Youssef Rochdi

ENT - Neck and Head surgery Department; University Medical Center Mohammed VI, Morocco.

(e-mail: Rochdi 86@ ${ }^{@}$ hotmail.com)

Hassan Nouri

ENT - Neck and Head surgery Department; University Medical Center Mohammed VI, Morocco.

(e-mail: Nouri_orl@yahoo.fr)

Abdelaziz Raji

ENT - Neck and Head surgery Department; University Medical Center Mohammed VI, Morocco.

(e-mail: Raji.abdelaziz ${ }^{@}$ gmail.com) problematic of this pathology lies in its latent character, it is, thus, much more frequent than we thought or predicted. Indeed, several studies have reported that the PTMC (Papillary tumor microcarcinoma) was detected up to $35.6 \%$ in the various autopsy series as latent carcinoma [3]. So, the main question remains shall we direct in those cases a minimalist attitude, being satisfied with the initial surgical gesture "the loboisthmectomy," or shall we be more maximalist advocating total excision of thyroid tissue. That is why, beyond specifying the epidemiological and clinical characteristics of papillary thyroid microcarcinoma, our ultimate goal was to determine the "gold standard" for the 
management of this entity, which although quite common is little known.

\section{TOOLS AND METHODOLOGY}

We carried out a descriptive retrospective study spread over 4 years from January 2014 to January 2018, including all cases of Papillary Microcarcinoma of the thyroid diagnosed within the department of ENT and Head and Neck Surgery of the medical center Mohammed VI in Marrakech; excluding all cases whose pathological diagnosis was unclear or doubtful. We collected 35 cases, and using their medical records we summarized their epidemiological, clinical and paraclinical data. We also studied the management of this entity and its prognosis.

\section{RESULTS}

The female gender, as in all studies concerning thyroid pathology, was largely predominant with a sex ratio of $5 / 1$; with 33 women for 2 men. The average age was $45+/-13.9$ years; with extremes of 31 years old for the youngest and 69 years old for the oldest, it is important to note that the predominant age group at $50 \%$ was 45 to 55 years old. To be more explicit, age over 45 accounted for more than $75 \%$ of our patients. Being a health system in Morocco of regional organization, most of our patients came from the region of Marrakech; this is by no means indicative of the endemic situation of this pathology within this region. Other locations were Safi and Errahmna. None of our patients had a family history of thyroid tumor disease, and only 5 of our patients were followed and treated for dysthyroidism, 3 cases of hyperthyroidism on synthetic antithyroidism treatment and 2 cases of hypothyroidism treated with Levothyroxine.

All our patients $(100 \%)$ had anterior cervical swelling beginning years before the first check-up with an average delay of consultation of 5 years, hence the importance of a systematic screening in Morocco and all over the world to diagnose early on cervical swelling. Three of our patients showed clinical signs of hyperthyroidism based on weight loss, tachycardia, chronic diarrhea and insidious anxiety. Some of our patients had compressive signs like dyspnea; dysphonia or dysphagia, without any case of superior vena cava syndrome (4 cases).

As we previously explained, none of our patients had a normal clinical examination, the palpation in all of our patients showed an isolated nodule in 7 patients or $20 \%$ of patients, the rest of our patients had clinically bilateral noticeable nodes. $80 \%$ of the nodes were not clinically suspicious, which mean they were mobile in relation to the deep plane, soft, not hard and not painful. We found cervical lymphadenopathy clinically palpable in three of our patients, two cases had recurrent projection lymphadenopathy on the right and the other bilateral, one case had superior cervical lymphadenopathy.

As part of the basic assessment due to thyroid nodes, all our patients benefited from a cervical ultrasound (Fig. 1) and all benefited from an EU-TIRADS classification for their nodes. The ultrasound was in favor of multi nodular goiter in 32 cases and isolated nodes in three patients including one case associated with an aspect of thyroiditis. In addition, cervical lymphadenopathy was objectified in three patients using ultrasound; as the clinical examination predicted. The majority of the nodes were solid (85\%), 15\% of the nodes were of mixed structure in the ultrasound. The main ultrasound character making the nodes suspicious was their hypoechoic character (10 cases), they were predominantly heterogeneous (15 cases). Microcalcifications were noted only in 6 patients. To conclude, according to the ultrasound data, a classification of our patients was made according to the EU-TIRADS classification. The number of patients with thyroid nodes classified as EU-TIRADS 3 was $45 \%, 28 \%$ were EU TIRADS 4; and $17 \%$ were EU TIRADS 5.

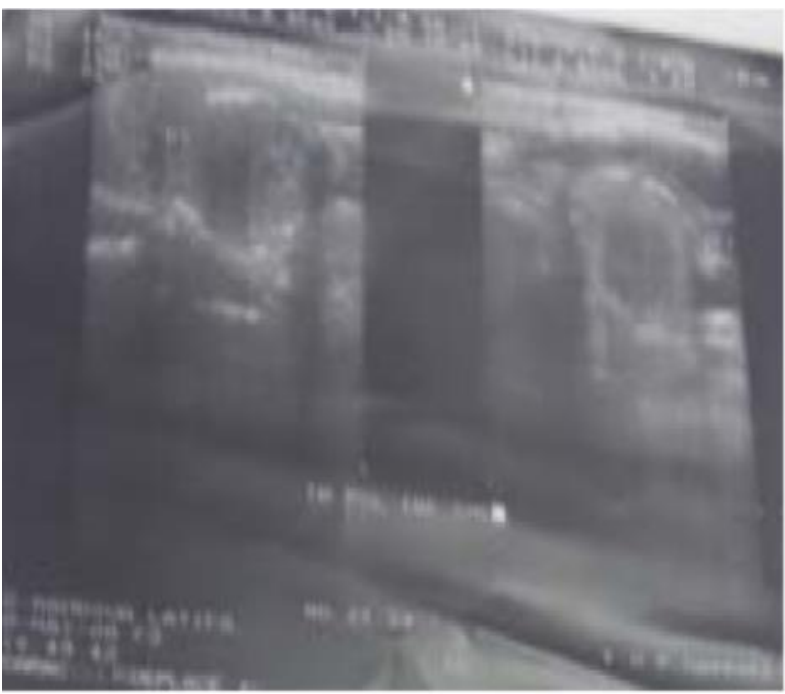

Fig.1. Thyroid node (Papillary microcarcinoma EU-TIRADS 3).

All our patients benefited from a hormonal status assessment including TSH us, T3 and T4, in the spirit of a biological study as comprehensive as possible, thus only 5 cases of dysthyroidism were found, including 2 cases of hypothyroidism and 3 others cases of hyperthyroidism. The rest of the patients had a normal hormonal status as attested by normal TSHus levels $(0.5-5 \mathrm{mU} / \mathrm{L})$. Scintigraphy was performed but in two cases (cases of patients with hyperthyroidism and a lone thyroid node), and objectified a hypo fixating left inferior polar node with homogeneous fixation of the tracer by the rest of the parenchyma. In the other case of hyperthyroidism, scintigraphy showed a slightly extinguishing node. The fine-needle aspiration biopsy did not allow the diagnosis in any case (it has reported cases of colloidal material pauci cell or hematic material without suspicious cells.); On the other hand, the ultrasound-guided fine-needle aspiration biopsy, which was much more efficient, allowed diagnosis in 3 cases, finding cellular material with nuclear abnormalities in favor of papillary carcinoma. No preoperative thyroglobulin dosage was performed in our series.

With regard to surgical treatment, 28 of our patients (80\%) underwent total thyroidectomy as a first step (Fig. 2), 4 patients underwent secondary totalization during the same operation after the extemporaneous examination came back 
positive for papillary microcarcinoma, 2 cases of remote loboisthmectomy after final histological examination showing papillary microcarcinoma, and one final case of totalization after extemporaneous examination of a superior right jugular lymph node with central necrosis. Neck dissection was performed in three cases. It focused on the left mediastinal-recurrent compartment in one patient, was bilateral of the same compartment in the other; and of the third and fourth jugular compartment in the last case. Ten patients had tumors less than $5 \mathrm{~mm}$ in size. The tumors sized between 5 and $10 \mathrm{~mm}$ was encountered in 20 of our patients. The average size of the papillary microcarcinoma was $5.36 \mathrm{~mm}$ with extremes ranging from $1.5 \mathrm{~mm}$ to $10 \mathrm{~mm}$. It was unifocal in 20 cases, multifocal and unilateral in 9 cases and multifocal, bilateral in 6 cases, none of which invaded the thyroid capsule. Anatomopathological examination of the lymph nodes taken during neck dissection revealed only one case of ganglionic metastases involving the upper right jugular group on the homolateral side of the tumor and two cases of reactive adenitis. There were no cases of distant metastasis in our series.

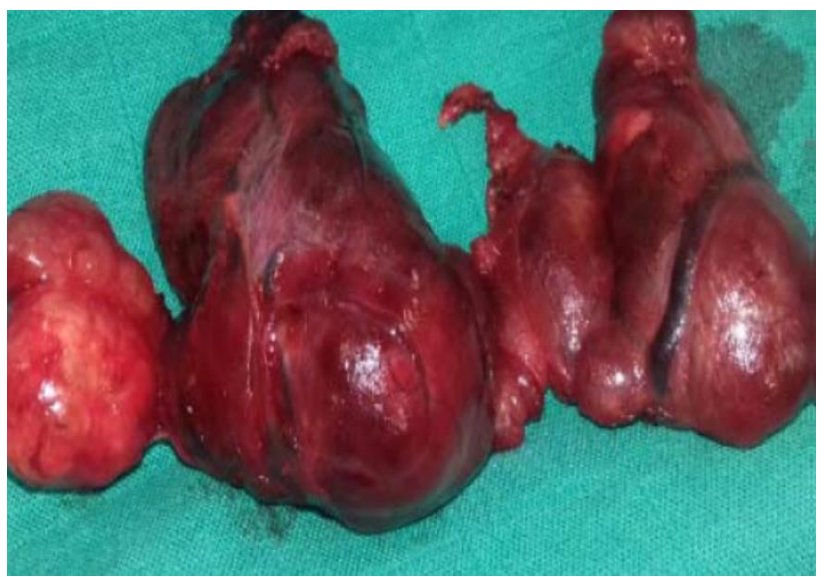

Fig. 2. Thyroid microcarcinoma (multinodular heterogenous goiter), total thyroidectomy.

Postoperative outcomes were simple in all patients. There was no case of iatrogenic hypoparathyroidism or postoperative dysphonia or cervical hematoma. A treatment based on antibiotics and analgesics was prescribed postoperatively to all of our patients. The removal of the drain was made on Day 2. The average duration of hospitalization was 2 days. Isotopic totalization by iodine 131 therapy was performed after 4 weeks of L-thyroxine withdrawal in four of our patients. Their indications were respectively: infiltrating tumor, extension to neighboring parenchyma, multifocality, lymph node metastasis. All four patients received a single course of treatment, with an oral dose of $100 \mathrm{mCi}(3.7 \mathrm{GBq})$. Isotopic scanning post-ira therapy was performed in all four cases. It did not objectify the presence of thyroid residues. Of the remaining 31, iratherapy was not indicated. These patients were classified as stage 1 with a very low risk of recurrence. Hormone replacement therapy was prescribed routinely after total thyroidectomy. All our patients were put on hormone therapy at physiological doses and then adapted after TSHus control. The control by TSHus was assured in all situations.
No patient received adjuvant chemoradiotherapy.

In order to watch for local or ganglionic recurrence and to adjust hormone replacement therapy, surveillance is a key part of the management, thus it was systematic in all cases as it should. It consisted of a clinical examination of the thyroid area with research of Cervical lymph nodes, the usual rhythm being one month, 3 months and then every 6 months. But also, the dosage of thyroglobulin and TSHus and an annual cervical ultrasound even without any suspicion of recurrence. The survival rate of our series was $100 \%$ (6 months to 4 years).

\section{DISCUSSION}

The World Health Organization monograph on the histological classification of thyroid tumors defines "Thyroid Papillary Micro Carcinoma" (MCPT) as "papillary cancer of $10 \mathrm{~mm}$ or less in its largest diameter". Currently, it is the most widely accepted definition, thus it is the definition we adopted in our study. This nomination has long been controversial and has been changed many times during recent years. These small sites of papillary carcinoma have been described under many names: "occult thyroid carcinoma", "latent papillary carcinoma", "small papillary carcinoma", "unencapsulated thyroid tumor", "occult sclerosing carcinoma", "microcarcinoma», "Micropapillary carcinoma". The maximum size of the lesion has not escaped controversy, ranging from $2.4 \mathrm{~mm}$ to $1.5 \mathrm{~cm}$ [4].

The incidence of papillary microcarcinoma is increasing in industrialized countries because of an earlier diagnosis and possibly an increase in the actual incidence of this disease. This increase could not be judged in the developing countries of which Morocco is a part but is most likely. It accounts for up to $30 \%$ of all differentiated thyroid carcinomas [5]. This high incidence therefore requires rapid and adequate management, or even systematic screening. Thyroid microcarcinoma affects all ages but has a peak of frequency for the 40 to 60 -year-old age group. Indeed, the frequency and risk of malignancy of a thyroid nodule increases with age. This was also noted in our study. The study of Belfiore and all and Mighri and all found a risk of malignancy respectively of $54 \%$ and $34.1 \%$ for subjects over 60 years of age [6]. Thus, age is obviously a factor of poor prognosis in this area. So, should we not be moving towards routine screening of thyroid nodes after a certain age as is already quite common for prostatic and breast cancer?

Studies showed that man has a higher risk of malignancy than woman [7], the high incidence of papillary microcarcinoma in women seems to be relative to the frequency of thyroid nodular pathology in the fairer sex. The literature is full of factors that can predispose to Papillary Microcarcinoma, even if none of its factors were found in our study, the most known and frequent are ionizing radiation, for example, the atomic bomb [7], even if this irradiation is accidental. The association between thyroid microcarcinoma and chronic lymphocytic thyroiditis has been described, but it is difficult to formally identify an etiological relationship. Genetics also plays a major role since recently, mutations of the BRAF gene (glutamic valine 
at residue 600) have been identified in about $45 \%$ of papillary microcarcinomas [8].

Thyroid papillary microcarcinoma can be discovered in a plethora of circumstances, whether it is screening for thyroid disease, or histological diagnosis during routine thyroid surgery or any other thyroid condition: multinodular heterogeneous goiter, Isolated node, Hyperthyroidism [8]. Generally the mean of discovery of this pathology tells us more about at least the diameter of the microcarcinoma, it is an average of $2.4 \mathrm{~mm}$ when the discovery is fortuitous and 5 $\mathrm{mm}$ when the patient has symptoms, such as the presence of a thyroid node whether it be suspicious or not, which remains the most common clinical form, such was the case in our study. These thyroid nodes exist in 4 to $7 \%$ of the general population, it is neither possible nor justified to operate all palpable thyroid nodes for the sole purpose of excluding malignancy. The investigations are intended to select for surgery patients whose cancer risk is high [9]. The thyroid node is highly suspected of malignancy when it is tender, firm or indurated, irregular or fixed, when there are signs of compression, or a rapid increase in size [9]. Remote metastases are rare in the presence of Microcarcinoma; their presence must evoke a vesicular carcinoma, more haematogenic than the papillary, although it is not to be definitively excluded; cases were found in the literature of distant metastasis in the case of Papillary Microcarcinoma [9].

Although rare, hyperthyroidism is possible in the context of papillary Microcarcinoma and justifies in our sense a systematic dosage of TSH us in these cases, beyond the diagnostic interest, it has, also, an anesthetic interest in the context of a possible surgical procedure. Most patients with normal serum TSH (0.5-5 mU/L) can go directly to the stage of ultrasound and needle aspiration biopsy, whereas patients with low serum TSH should be conducting morphological and scintigraphy examinations [9].

Ultrasound is the exam of choice for thyroid imaging far superior to cervical CT and cervical MRI, those exams rarely find their place in the management of this entity apart from the assessment of the extension of some extensive forms. It is relatively inexpensive, easily accessible, quickly executed and innocuous. It is, however, dependent on the radiologist's interpretation. It is currently recommended for all patients with thyroid nodes [10]. Unfortunately, the popularization of ultrasound has led to an increase in the proportion of T1-2N0M0 stages, from $57.4 \%$ (in 1991) to $70.6 \%$ (in 2000) [10]. Thus, should the use of ultrasound be limited to certain cases in which the clinical examination is in favor of a papillary microcarcinoma or systematic in every case of thyroid node? Studies have shown that certain ultrasound characteristics increase the relative risk (RR) of cancer in thyroid nodes: hypoechoic character (RR 1.5 to 3.0), fuzzy limits (RR 1.1 to 6.0), microcalcifications (RR 2.0 to 5.0 ), intra-nodular hyper-vascularization ( $R R, 4.0$ to 5.0 ), or a wider than a high diameter on a cross-section (RR 2.0 to 2,5$)$ [11]. It is the EU-TIRADS classification that sums up these criteria quite well and which, by advancing in the stages, increases the tumor risk of the node. This allowed the evaluation of the risk of malignancy and allowed to standardize the management to be held. Beyond the ultrasound characteristics of a thyroid node, cervical ultrasound can also participate in the exploration of regional lymph nodes, and to testify to their nature, because of its high sensitivity 90 to $92 \%$. [11] Characteristics that may indicate malignancy include a rounded shape, an unrecognizable hilum, an eccentric enlargement of the cortex, fuzzy boundaries, hypo echogenicity or heterogeneous echo structure, and an alteration of intraganglionic vascularization: hyper vascularization or anarchic vascular network; cystic areas that partially or totally reach the ganglion, and calcifications [11].

Scintigraphy has a low specificity and positive predictive value for detecting cancer (about 10-15\%), [12] and is much less efficient than needle aspiration biopsy to determine tumor risk [13]. Its only interest, really, in the context of thyroid pathology, is a single thyroid node with a low TSHus, even if these hyperfixing nodes are almost never malignant. The needle aspiration biopsy is intended for all palpable suspicious nodes. For non-palpable nodes, it is recommended to tap only supra-centimeter, or from $8 \mathrm{~mm}$ only if the clinical context is suggestive and / or if the node is solid and hypoechoic [13]. In the case of multinodular goiter, the suspicious and then the larger nodes will be first and foremost punctured. Ultrasound-guided aspirationneedle biopsy is much more efficient since it allows to aim and to puncture a specific suspicious node. This is an inexpensive, fast exam with few or no complications. Some authors have suggested that on the basis of cytology one can differentiate between Microcarcinoma and papillary carcinoma. The microcarcinoma shows only a few groups of papillary-like cells in a fundus of benign follicular cells and by repeating the aspirations of this same node will be devoid of any suspicious cytology [13].

During the last two decades, the surgical management of well-differentiated thyroid carcinoma has been the subject of controversies in order to determine the most appropriate one. These controversies concern macroscopic carcinoma as well as microscopic carcinoma or MCT.

Thus, there are two opposing attitudes: that of the minimalists who, in the presence of a thyroid microcarcinoma, are satisfied with a loboisthmectomy and a surveillance in search of a locoregional recurrence versus a maximalist attitude which proposes on the contrary a total thyroidectomy and a variable lymph node surgery comprising at least ipsilateral recurrence dissection, as well as ira-therapy according to the results of the postoperative scintigraphy [13].

It is important, in order to decide between these two schools, to balance the surgical risks of total thyroidectomy (vital, bilateral recurrent paralysis, hypothyroidism and hypoparathyroidism) and the fact that the papillary microcarcinoma of the thyroid has a benign biological profile , that it is usually multifocal with a histopathological expression but, with little clinical expression and small sites left in place may even regress, there is also a low risk of local recurrence and distant metastases are exceptional [13].

Regarding the indications of lymph nodes dissection, two tendencies contrast, one advocating for lymph node 
dissection more or less extended, the other one advocating for the removal of only invaded lymph nodes proved clinically, instrumentally or during intraoperative examination. For most authors, picking or berry picking techniques, that is, the removal of suspect lymph nodes only, have no sufficient diagnostic value or therapeutic role. They must be proscribed because of the importance of locoregional recurrences of up to $33 \%$ [14]. Microscopic lymph node metastases are frequent in the MCPT, the lymphadenectomy of principle would show 70 to $80 \%$ of lymphatic metastases [14]. However, prophylactic lymph node dissection would seem unnecessary [14].

Hormone replacement therapy is routine after total or near total thyroidectomy. It is estimated that after conservative treatment, inhibitory hormone therapy slows down the evolution of microsites left in place. However, the interest of a treatment blocker in the therapeutic management of thyroid microcarcinoma is not demonstrated. Despite this, all our patients are put on hormone therapy at supra physiological doses given the hormone-dependent nature of MCT [16].

The surveillance of these patients is not only clinical, it is also based on ultrasound and biology, and is one of the cornerstones of the management of this pathology. The cervical palpation is thus systematic in spite of not being very sensitive and operator dependent. Cervical ultrasound; as for it, has become the key examination of thyroid cancer surveillance. Initially, clinical monitoring is performed on a monthly basis for 3 months, then it is semi-annual for 2 years and then becomes annual. At present, regular dosages of TSH and thyroglobulin prevent repeated scintigraphy. Only when the thyroglobulin is increased will whole body scintigraphy be performed after one month of discontinuation of hormone replacement therapy [17].

Thyroid microcarcinomas are generally of good prognosis, long-term survival is generally favorable, but for some patients there is a risk of relapse. Disease-specific mortality rates have been reported at less than $1 \%$, with locoregional recurrence rates of 2-6\% [17]. Older age, male sex, histological type, tumor size $>5 \mathrm{~mm}$, presence of lymph node metastasis, multifocality, distant metastases, and extrathyroid invasion are all factors of poor prognosis [17].

\section{CONCLUSION}

Papillary Microcarcinoma, a histological type of papillary carcinoma limited by the size of less than one $\mathrm{cm}$, is a tumor with a better prognosis than its counterpart. Their generally silent nature makes their diagnosis difficult and is usually a silent entity. The therapeutic protocol is punctuated with controversy, and faces a conservative attitude versus a radical one, it is the surgeon's duty to distinguish between the two attitudes.

\section{CONSENT}

Oral consent of the patients was obtained for the publication of these cases.

\section{REFERENCES}

[1] M. M. Touati and al., Les carcinomes de la thyroïde : profils épidémiologique, clinique et thérapeutique, à propos de 102 cas," Pan Afr. Med. J., vol. 21, no. 1, 2015.

[2] R. Ghossein, I. Ganly, A. Biagini, E. Robenshtok, M. Rivera, and R. M. Tuttle, "Prognostic factors in papillary microcarcinoma with emphasis on histologic subtyping: a clinicopathologic study of 148 cases," Thyroid, vol. 24, no. 2, pp. 245-253, 201.

[3] M.Moumen, M.Touhami, M.E.Elaloui, F.El.Fares. « Les microcarcinomes thyroidiens » Médecine du Maghreb 1992 n³1.

[4] Yamashita H, Noguchi S, Watanabe S et al. Thyroid cancer associated with adenomatous goiter: an analysis of the incidence and clinical factors. Surgery Today 1997; 27: 495-499.

[5] Yu XM,Wan Y, Sippel RS et al. Should all papillary thyroid microcarcinomas be aggressively treated? An analysis of 18,445 cases. Annals of Surgery 2011; 254: 653-660.

[6] Chow SM, Law SC, Chan JK et al. Papillary microcarcinoma of the thyroid. Prognostic significance of lymph node metastasis and multifocality. Cancer 2003; 98: 31-40.

[7] Baudin E, Travagli JP, Ropers J et al. Microcarcinoma of the thyroid gland. The Gustave-Roussy Institute experience. Cancer 1998; 83: 553-559.

[8] Bozec A, Dassonville O, Chamorey E, et al. Clinical impact of cervical lymph node involvement and central neck dissection in patients with papillary thyroid carcinoma: a retrospective analysis of 368 cases. Eur Arch Otorhinolaryngol 2011; 268:1205-12.

[9] Pacini F, Schlumberger M, Dralle H, et al. European consensus for the management of patients with differentiated thyroid carcinoma of the follicular epithelium. Eur J Endocrinol 2006; 154:787-803.

[10] Lamhamedi A. Le point sur les microcarcinomes . Maghreb médical, 1985, 113: 29-38.

[11] Ito $\mathrm{Y}$, Miyauchi A, Inoue $\mathrm{H}$ et al. An observational trial for papillary thyroid microcarcinoma in Japanese patients. World Journal of Surgery $2010 ; 34: 28-35$.

[12] Ito Y, Uruno T, Nakano K et al. An observation trial without surgical treatment in patients with papillary microcarcinoma of the thyroid. Thyroid 2003; 13: 381-387.

[13] Baudin E, Travagli Jp, Ropers J, Mancusi F, Bruno Bossio G, Caillou B, Cailleux Af, Lumbroso Jd, Parmentier C, Schlumberger M: Microcarcinoma of the thyroid gland. Cancer, 1998; 83: 553-558

[14] G. Sturniolo, M. G. Lo. Schiavo, C. D'alia, A. Tonante, E. Gagliano, F. Tarano, 1. Bonanno, G. Sturniolo: Le traitement chirurgical des microcarcinomes thyroïdiens. E-mémoires de l'Academie Nationale de Chirurgie, 2004, 3 (1): 47-52.

[15] British Thyroid Association and Royal College of Physicians Guidelines for the management of thyroid cancer in adults. London: RCP, 2002.

[16] Hay Ian D, Grant Cs, Herden Jav, Goellner Jr, Ebersold Jr, Bergstralh Ej; Papillary thyroid microcarcinoma: a study of 535 cases observed in 50-year period. Surgery, 1992;113:1139-47.

[17] Rossi Rl, Cady Ml, Silverman Ml, Wool Ms, Horner Ta. Current results of conservative surgery for differenciated thyroid carcinoma. World J Surg 1986; 10:612-622. 